5. A. Rényi, On the density of certain sequences of integers, Acad. Serbe Sci. Publ. Inst. Math. 8 (1955), 157-162.

6. R. Robinson, An infinite product factorization for a class of generating functions defined by power series, Ph.D. dissertation, Univ. of Tennessee, Knoxville, Tenn., 1964.

UNIVERSITY OF TENNESSEE

\title{
ANTIFLEXIBLE ALGEBRAS WHICH ARE NOT POWER-ASSOCIATIVE
}

\section{J. RODABAUGH}

1. Introduction. An algebra $A$ will be called antiflexible if the elements of $A$ satisfy the identity

$$
(x, y, z)=(z, y, x) \text {. }
$$

Power-associative antiflexible algebras have been studied by the author [3] and Kosier [2].

As a matter of terminology, we shall define an algebra as a finite dimensional vector space on which a multiplication is defined in which both distributive laws are satisfied. If $A$ is an algebra over a field $F$ of characteristic not two, then $A$ has an attached algebra $A^{+}$ which is the same additive group as $A$ but the multiplication $x \cdot y$ of $A^{+}$is defined by

$$
x \cdot y=(x y+y x) / 2 .
$$

In any algebra $x^{n}$ is inductively defined by $x^{1}=x$ and $x^{k}=x^{k-1} x$. An algebra is power-associative if $x^{a} x^{b}=x^{a+b}$ for all $a, b$. The associator $(x, y, z)$ is defined as $(x y) z-x(y z)$ and the commutator $(x, y)$ is defined as $x y-y x$. For our purposes, $A$ is nodal if every member of $A$ can be written as $\alpha \cdot 1+z$ with $z$ nil in $A^{+}$.

Since we are not assuming power-associativity, we will define the radical as the maximal ideal of $A$ which is nil in $A^{+}$. An algebra is semisimple if its radical is zero. An algebra is simple if it is semisimple and if it contains no proper ideals. It should be clear that, in the case of power-associative antiflexible algebras, these definitions are equivalent to the ones in [2], [3].

\footnotetext{
Presented to the Society, August 31, 1964; received by the editors October 22,
} 1964. 
In $\S 2$ we will construct for each prime $p \neq 0,2$ a simple, nodal, $p$-dim. antiflexible algebra $A_{p}$ of char. $p$ which satisfies the following conditions:

$$
\begin{aligned}
x^{a} x^{b} & =x^{a+b} \quad \text { for } a+b<p \text { and } x \text { in } A_{p} \\
x^{a} x^{p-a} & \neq x^{p-a} x^{a}
\end{aligned}
$$

It is known that when the characteristic is zero, an antiflexible algebra that satisfies $(x, x, x)=0$ is power-associative [2].

In $\$ 3$ remarks are made that extend known structure theorems to antiflexible algebras that satisfy $(x, x, x)=0$.

2. Construction and properties of $A_{p}$.

Definition 1. Given a field $F$ of characteristic $p \neq 0,2$; consider the vector space $V_{p}$ spanned by $1, x, \cdots, x^{p-1}$ over $F$. The algebra $A_{p}$ will be defined as this vector space with multiplication determined by the following:

$$
\begin{aligned}
x^{a} x^{b} & =x^{a+b} \quad \text { if } a+b \leqq p-1 \\
x^{a} x^{b} & =0 \quad \text { if } a+b>p \\
x^{p-a} x^{a} & =a \alpha \cdot 1 \quad \text { where } \alpha \text { is a fixed nonzero element in } F .
\end{aligned}
$$

To prove that $A_{p}$ is antiflexible, we first observe that the associator $\left(x^{a}, x^{b}, x^{c}\right)$ can have the following values:

(a) 0 when $a+b+c<p$,

(b) 0 when $a+b+c>p, a+b \neq p$, and $b+c \neq p$,

(c) $-b \alpha \cdot 1$ when $a+b+c=p$,

(d) $-c \alpha x^{a}$ when $a+b+c>p, a+b \neq p$ and $b+c=p$,

(e) $b \alpha x^{c}$ when $a+b+c>p, b+c \neq p$ and $a+b=p$,

(f) $b \alpha x^{c}-c \alpha x^{a}$ when $a+b+c>p, a+b=p$ and $b+c=p$.

The antiflexible law clearly holds in the cases represented by (a), (b) and (c). In the case represented by (d), $\left(x^{c}, x^{b}, x^{a}\right)=b \alpha x^{a}=(p-c) \alpha x^{a}$ $=-c \alpha x^{a}=\left(x^{a}, x^{b}, x^{c}\right)$. In a similar fashion $\left(x^{a}, x^{b}, x^{c}\right)=\left(x^{c}, x^{b}, x^{a}\right)$ in the other cases. Therefore, $A_{p}$ is antiflexible.

Suppose $w=a_{0} \cdot 1+\sum_{i=1}^{p-1} a_{i} x^{i} \neq 0$ and suppose $w$ is in an ideal $J$ of $A_{p}$. If $k$ is the largest integer with $a_{k} \neq 0$ then $x^{p-k} w=k \alpha a_{k} \cdot 1$ $+\sum_{i=1}^{k-1} a_{i} x^{i+p-k}$ and $w x^{p-k}=(p-k) \alpha a_{k} \cdot 1+\sum_{i=1}^{k-1} a_{i} x^{i+p-k}$. Therefore $\left(x^{p-k}, w\right)=2 k \alpha a_{k} \cdot 1$ is in $J$ so, for $y$ in $A_{p}, y=\left(y /\left(2 k \alpha a_{k}\right)\right)\left(x^{p-k}, w\right)$ is in $J$. Hence, $A_{p}$ is simple. We have proved the following lemma.

LeмMA 1. The algebra $A_{p}$ is antiflexible and simple.

Theorem 1. The algebra $A_{p}$ is simple, nodal, p-dimensional, antiflexible, and for an arbitrary $y$ in $A$, we have $y^{a} y^{b}=y^{a+b}$ for $a+b<p$. Furthermore, $A_{p}$ is generated by one element $x$ and $x^{p-a} x^{a} \neq x^{a} x^{p-a}$. 
PROOF. Every part of this is clear except for the fact that $y^{a} y^{b}$ $=y^{a+b}$ for $a+b<p$. First, if $(y, y, y)=0$ then this can be shown as follows: From Albert [1], if $n<p$ we immediately find that $\left(y^{n-1}, y\right)$ $=0$. In [2] it was shown that in an antiflexible algebra with $(y, y, y)$ $=0, y^{a+b}=y^{a} y^{b}$ for $a+b=4$. Consequently, from [1], $y^{a+b}=y^{a} y^{b}$ if $a+b<p$. If $p=3$, the result is trivial so we need only show that $(y, y, y)=0$ when characteristic $\neq 3$ and the result is established. Linearization of $(y, y, y)=0$ gives

$$
(u, v, w)+(v, w, u)+(w, u, v)+(w, v, u)+(v, u, w)+(u, w, v)=0 .
$$

Since $A_{p}$ is antiflexible and since the characteristic is not 2 , we have

$$
(u v, w)+(v w, u)+(w u, v)=(u, v, w)+(v, w, u)+(w, u, v)=0 .
$$

Therefore, our proof is complete when we show that (9) holds for basis elements of $A_{p}$. If any of $u, v$ or $w$ is 1 , (9) holds so let $u=x^{a}$, $v=x^{b}$ and $w=x^{c}$ and let $n=a+b+c$. We then have

$$
\left(x^{n-c}, x^{c}\right)+\left(x^{n-a}, x^{a}\right)+\left(x^{n-b}, x^{b}\right)=0 .
$$

If $n \neq p,(10)$ is obviously satisfied by Definition 1 . For $n=p$, we have $2 c \alpha \cdot 1+2 a \alpha \cdot 1+2 b \alpha \cdot 1$ which is 0 .

3. The structure of antiflexible algebras with $(x, x, x)=0$. With only slight changes, the structure theorems in [2] and [3] can be proved for antiflexible algebras with $(x, x, x)=0$. The only identities used to prove these theorems are $(x, y, z)=(z, y, x)$ and $(x, x, x)=0$ or linearizations of these identities. Power-associativity was only used to guarantee the existence of idempotents in simple and semisimple algebras. This can be accomplished with our definitions of simple and semisimple by noting that if $A$ is antiflexible and satisfies $(x, x, x)=0$ then $A^{+}$is Jordan. Hence, $A^{+}$is power-associative. If $A$ is semisimple then there exists an idempotent $e$ in $A^{+}$. Clearly, $e$ is an idempotent in $A$.

\section{BIBLIOGRAPHY}

1. A. A. Albert, Power-associative rings, Trans. Amer. Math. Soc. 64 (1948), 552597.

2. Frank Kosier, On a class of nonflexible algebras, Trans. Amer. Math. Soc. 102 (1962), 299-319.

3. D. Rodabaugh, $A$ generalization of the flexible law, Trans. Amer. Math. Soc. 114 (1965), 468-487.

VANDERBILT UNIVERSITY 